\title{
Prediction of slope failures using bivariate statistical based index of entropy model
}

\begin{abstract}
The main objective of this research is to evaluate the spatial prediction of potential slope failures in Kuala Lumpur and surrounding areas using an index of entropy based statistical model. Based on potential information of entropy method (IoE), subjective weights were calculated for fourteen landslide conditioning factors used in this study such as, (slope, aspect, curvature, altitude, surface roughness, lithology, distance from faults, NDVI (normalized difference vegetation index), land cover, distance from drainage, distance from road, SPI (stream power index), soil type and precipitation). A landslide inventory map of the study area was produced using previous reports and aerial photographs interpretation aided with extensive field survey and total of 220 main scarps were identified. Out of this, 153 (70\%) landslide locations were used to build the IoE model, while remaining 66 (30\%) landslide locations were used for validation purpose. For validation, the area under the curve (AUC) was used to quantify the predictive performance of the employed IoE model. The validation results show that the prediction accuracy of the model is $0.80(80 \%)$ and the success rate equals to $0.81(81 \%)$ that consider fine indicator of the reliability of bivariate model based IoE model employed in this study.
\end{abstract}

Keyword: Landslides; Kuala Lumpur; Bivariate model; Index of entropy; Geographic information systems (GIS); Remote sensing 